\title{
Correction to: Impact of Ipsilateral Supraclavicular Lymph Node Dissection (ISLND) for Breast Cancer Patients and a Nomogram for Predicting Ipsilateral Supraclavicular Pathological Complete Response (ispCR)
}

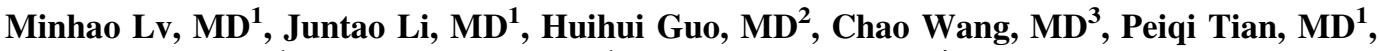 \\ Youzhao Ma, MD ${ }^{1}$, Xiuchun Chen, $\mathrm{MD}^{1}$, and Suxia Luo, $\mathrm{MD}^{4}$ \\ ${ }^{1}$ Department of Breast Disease, The Affiliated Cancer Hospital of Zhengzhou University, Henan Cancer Hospital, \\ Zhengzhou, China; ${ }^{2}$ Department of General Surgery, Jiyuan People's Hospital, Jiyuan, China; ${ }^{3}$ Department of General \\ Surgery, Huaxian People's Hospital, Huaxian, China; ${ }^{4}$ Department of Medical Oncology, The Affiliated Cancer Hospital of \\ Zhengzhou University, Henan Cancer Hospital, Zhengzhou, China
}

\section{CORRECTION TO: ANN SURG ONCOL HTTPS://DOI.ORG/10.1245/S104 34-020-09548-6}

The article Impact of Ipsilateral Supraclavicular Lymph Node Dissection (ISLND) for Breast Cancer Patients and a Nomogram for Predicting Ipsilateral Supraclavicular Pathological Complete Response (ispCR), written by Lv et al., was originally published online on the publisher's internet portal on February 17, 2021, with Open Access under a Creative Commons Attribution (CC BY) license 4.0. With the authors' decision to cancel Open Access the copyright of the article changed on March 3, 2021 to (C) Society of Surgical Oncology 2021 with all rights reserved.

The original article has been updated.

Publisher's Note Springer Nature remains neutral with regard to jurisdictional claims in published maps and institutional affiliations.

The original article can be found online at https://doi.org/10.1245/ s10434-020-09548-6.

(C) Society of Surgical Oncology 2021

Published Online: 18 March 2021

S. Luo, MD

e-mail: zlyyluosuxia0361@zzu.edu.cn 